\title{
Model-reduction in micromechanics of polycrystalline materials
}

\author{
Jean-Claude Michel $^{1}$ and Pierre Suquet ${ }^{1, *}$ \\ ${ }^{1}$ Aix Marseille Univ, CNRS, Centrale Marseille, LMA, 4 impasse Nikola Tesla, \\ CS 40006, 13453 Marseille Cedex 13, France
}

\begin{abstract}
The present study is devoted to the generalization of the Nonuniform Transformation Field Analysis (NTFA), a model-reduction approach introduced by the authors. First, the local fields of internal variables are decomposed on a reduced basis of modes. Second, the effective (average) dissipation potential of the phases is replaced by accurate approximations. The reduced evolution equations of the models, in other words the homogenized constitutive relations, can be entirely expressed explicitly in terms of quantities which are pre-computed "off-line". The example of creep of polycrystalline ice is used to assess the accuracy of the models. Their predictions, both the overall response and the local response, are shown to be in good agreement with full-field simulations with a significant speed-up.
\end{abstract}

\section{Reduced-order models}

A common engineering practice in the analysis of composite (or polycrystalline) structures is to use effective or homogenized material properties instead of taking into account all details of the individual phase properties. Unfortunately when the individual constituents are nonlinear, the exact description of the effective constitutive relations requires the determination of the local fields (at the microscopic scale). For structural computations, the consequence of this theoretical result is that the two levels of computation, the level of the structure and the level of the unit-cell, remain intimately coupled. The nested resolution of these coupled problems (known as $\mathrm{FE}^{2}$ analysis) is so far limited by their formidable size.

It is therefore quite natural to resort to model-reduction techniques achieving a compromise between analytical approaches, which are costless but often very limited by nonlinearity, and full-field simulations which resolve all complex details of the exact solutions, but come at a very high cost. This is the aim of reduced-order modelling (ROM) in general $([1,2])$. However, ROM is often understood

\footnotetext{
*Corresponding author: e-mail suquet@lma.cnrs-mrs.fr, phone +33484524296
} 
as a way to reduce the cost of a numerical simulation. In homogenization problems, the objective is not only to reduce the computational cost, but also to arrive at an explicit constitutive model, where internal variables are identified and evolution equation for these internal variables are explicitly derived. The model which is reduced is not only the computational model, but also and primarily, the constitutive model.

General procedures to achieve the model reduction do exist, at least for the computational model. However, without a proper physical insight in the problem to be reduced, it is likely that these general procedures will not deliver satisfactory answers. The model initially proposed by the authors ( [3]) and further developed in [7-9] and by other authors $([4,5])$ is limited in scope to materials with a microstructure, comprised of constituents with a certain type of constitutive relations involving internal variables. In turn, the approximations on which the model is based are physically sound in this context. The three main ingredients of the model are as follows.

1. Experiments and numerical simulations evidence the patterning of the local fields induced by the presence of a microstructure. This patterning reveals the existence of a few microscopic mechanisms by which the composite deforms.

2. When the mechanical response of the individual constituents is governed by two potentials, the free-energy potential corresponding to reversible effects and the dissipation potential corresponding to dissipative effects, the overall response of the composite is also governed by two effective potentials which are the average of the local potentials. However deriving an exact expression for these two potential is a difficult task, which cannot be achieved in a closed form when the constituents are nonlinear.

3. Recognizing that the problem of finding an averaged potential is similar to that encountered in homogenization of nonlinear composites with a single potential ( [10]), it is quite natural to transpose some of the techniques of nonlinear homogenization to the present context of ROM.

\subsection{Individual constituents}

The composite (or polycrystalline) materials considered in this study are comprised of individual constituents undergoing partly reversible and partly irreversible transformations modelled by a finite number of internal variables $\boldsymbol{\alpha}$. It is further assumed that the stress derives from a free-energy function $w(\varepsilon, \boldsymbol{\alpha})$ and that the evolution of the variables $\boldsymbol{\alpha}$ is governed by the driving forces associated with $\boldsymbol{\alpha}$. It is often the case (but not always) that this last relation can be expressed with the help of a dissipation potential $\varphi$ and the constitutive relations take the compact form,

$$
\boldsymbol{\sigma}=\frac{\partial w}{\partial \varepsilon}(\varepsilon, \boldsymbol{\alpha}), \quad \frac{\partial w}{\partial \boldsymbol{\alpha}}(\varepsilon, \boldsymbol{\alpha})+\frac{\partial \varphi}{\partial \dot{\boldsymbol{\alpha}}}(\dot{\boldsymbol{\alpha}})=0 .
$$

\subsection{Effective potentials}

A representative volume element (r.v.e.) $V$ of the material is comprised of phases occupying domains $V^{(r)}$. The spatial averagings over $V$ and $V^{(r)}$ are respectively denoted by $\langle$.$\rangle and \langle.\rangle^{(r)}$. Each individual phase is governed by the above differential equation with potentials $w^{(r)}$ and $\varphi^{(r)}$ in $V^{(r)}$. The 
r.v.e. $V$ is subjected to a path of macroscopic strain $\bar{\varepsilon}(t)$ and periodicity conditions on $\partial V$. The local problem to be solved to determine the local fields $\boldsymbol{\sigma}(\boldsymbol{x}, t), \boldsymbol{\varepsilon}(\boldsymbol{x}, t)$ and $\boldsymbol{\alpha}(\boldsymbol{x}, t)$ consists of a generalized thermoelastic problem (2), in which the field of internal variables $\boldsymbol{\alpha}(\boldsymbol{x})$ is fixed,

$$
\left.\begin{array}{c}
\boldsymbol{\sigma}(\boldsymbol{x}, t)=\frac{\partial w}{\partial \boldsymbol{\varepsilon}}(\boldsymbol{x}, \boldsymbol{\varepsilon}(\boldsymbol{x}, t), \boldsymbol{\alpha}(\boldsymbol{x}, t)), \quad \operatorname{div} \boldsymbol{\sigma}(\boldsymbol{x}, t)=0, \\
\boldsymbol{\varepsilon}(\boldsymbol{x}, t)=\overline{\boldsymbol{\varepsilon}}(t)+\frac{1}{2}\left(\boldsymbol{\nabla} \boldsymbol{u}^{*}(\boldsymbol{x}, t)+\boldsymbol{\nabla} \boldsymbol{u}^{* T}(\boldsymbol{x}, t)\right), \quad \boldsymbol{u}^{*} \text { periodic on } \partial V, \\
\boldsymbol{\sigma} \cdot \boldsymbol{n} \text { anti-periodic on } \partial V,
\end{array}\right\}
$$

coupled with the differential equation $\left(1_{2}\right)$ at every point $\boldsymbol{x}$ in the volume element. The homogenized (or effective) response of the composite along the path $\{\overline{\boldsymbol{\varepsilon}}(t), t \in[0, T]\}$ is the history of average stress $\{\overline{\boldsymbol{\sigma}}(t), t \in[0, T]\}$ where $\overline{\boldsymbol{\sigma}}(t)=\langle\boldsymbol{\sigma}(\boldsymbol{x}, t)\rangle$. It can be shown that the overall response of the composite is governed by two potentials

$$
\overline{\boldsymbol{\sigma}}=\frac{\partial \widetilde{w}}{\partial \bar{\varepsilon}}(\bar{\varepsilon}, \boldsymbol{\alpha}), \quad \frac{\partial \widetilde{w}}{\partial \boldsymbol{\alpha}}(\bar{\varepsilon}, \boldsymbol{\alpha})+\frac{\partial \widetilde{\varphi}}{\partial \dot{\boldsymbol{\alpha}}}(\dot{\boldsymbol{\alpha}})=0, \quad \widetilde{w}(\bar{\varepsilon}, \boldsymbol{\alpha})=\operatorname{Inf}_{\langle\varepsilon\rangle \overline{\boldsymbol{\varepsilon}}}\langle w(\varepsilon, \boldsymbol{\alpha})\rangle, \quad \widetilde{\varphi}(\dot{\boldsymbol{\alpha}})=\langle\varphi(\dot{\boldsymbol{\alpha}})\rangle,
$$

where unfortunately $\boldsymbol{\alpha}$ is a field (infinitely many internal variables).

\subsection{Reduced variables and reduced potentials}

In order to reduce the number of macroscopic internal variables, and inspired by numerical simulations and experiments which clearly show the patterning of the plastic strain field in the volume element, the Nonuniform Transformation Field Analysis (NTFA) proposed by Michel and Suquet [3] is based on a decomposition of the fields of internal variables on a finite set of pre-determined shape functions (NTFA decomposition)

$$
\boldsymbol{\alpha}(\boldsymbol{x}, t)=\sum_{k=1}^{M} \xi^{(k)}(t) \boldsymbol{\mu}^{(k)}(\boldsymbol{x}),
$$

where the fields $\boldsymbol{\mu}^{(k)}(\boldsymbol{x})$ are the modes and the $\xi^{(k)}$ are the reduced variables. The modes have the same tensorial character as the internal variables $\boldsymbol{\alpha}$. To avoid a possible indeterminacy in the definition of the reduced variables $\xi^{(k)}$, it is further assumed that the modes $\boldsymbol{\mu}^{(k)}$ are linearly independent fields. How the modes are generated is recalled in section 1.4. Using the decomposition (4) into the variational definition (3) of the two effective potentials, an approximation of the evolution equations for the reduced variables $\boldsymbol{\xi}$ is given by

$$
\overline{\boldsymbol{\sigma}}=\frac{\partial \widetilde{w}}{\partial \bar{\varepsilon}}(\overline{\boldsymbol{\varepsilon}}, \boldsymbol{\xi}), \quad \frac{\partial \widetilde{w}}{\partial \boldsymbol{\xi}}(\overline{\boldsymbol{\varepsilon}}, \boldsymbol{\xi})+\frac{\partial \widetilde{\varphi}}{\partial \dot{\boldsymbol{\xi}}}(\dot{\boldsymbol{\xi}})=0, \quad \widetilde{w}(\overline{\boldsymbol{\varepsilon}}, \boldsymbol{\xi})=\operatorname{Inf}_{\langle\boldsymbol{\varepsilon}\rangle=\overline{\boldsymbol{\varepsilon}}}\langle w(\varepsilon, \boldsymbol{\alpha}(\boldsymbol{\xi}))\rangle, \quad \widetilde{\varphi}(\dot{\boldsymbol{\xi}})=\langle\varphi(\dot{\boldsymbol{\alpha}}(\dot{\boldsymbol{\xi}}))\rangle,
$$

where $\boldsymbol{\alpha}(\boldsymbol{\xi})$ is given by the NTFA decomposition (4). In many cases of interest, the effective freeenergy $\widetilde{w}$ can be expressed in terms of pre-computed quantities and the difficulty lies in the evaluation of the effective dissipation potential $\widetilde{\varphi}$ in terms of $\dot{\xi}$ without computing "on-line" the local fields $\dot{\alpha}$. 


\subsection{Snapshot POD}

The modes $\boldsymbol{\mu}^{(k)}(\boldsymbol{x})$ are generated by the snapshot Proper Orthogonal Decomposition (POD) method along some training loading paths, whose choice depends heavily on the application that the user has in mind ( [6]). Along a given training path, $T$ snapshots of the appropriate internal variable fields computed by the full-field method are stored at different time steps. Then, following the classical POD procedure, the correlation matrix $\boldsymbol{g}$ of these snapshots is formed and its eigenvalues $\lambda^{(k)}$ and eigenvectors $\boldsymbol{v}^{(k)}$ are computed. The actual modes are expressed as a combination of the eigenvectors as

$$
g^{(k \ell)}=\left\langle\boldsymbol{\theta}^{(k)}: \boldsymbol{\theta}^{(\ell)}\right\rangle, \quad \boldsymbol{\mu}^{(k)}(x)=\sum_{\ell=1}^{T} v_{\ell}^{(k)} \boldsymbol{\theta}^{(\ell)}(x) .
$$

A main interest in the snapshot POD method lies in the fact that the quantity of relevant information (its correlation with the set of snapshots) contained in an eigenvector $\boldsymbol{v}^{(k)}$ is expressed by the magnitude of the corresponding eigenvalue $\lambda^{(k)}$. This property is used to truncate the set of modes, retaining the most relevant ones ( [6]).

\subsection{Approximate effective potentials and nonlinear homogenization}

The "reduced kinetic" equations of the model consist of (5). In order to arrive at a closed form expression of these equations one has to compute explicitly the effective potentials $\widetilde{w}$ and $\widetilde{\varphi}$. This is the most difficult part of the procedure which requires additional physical and mathematical simplifications.

The first simplification is that the free-energy function $w$ of the individual constituents is assumed to be quadratic with respect to $\varepsilon$ and $\boldsymbol{\alpha}$ (when this is not the case a work-around has been proposed in [7] and [8]). Then the thermoelastic problem (2) becomes linear, its solution can be expressed by superposition as

$$
\boldsymbol{\sigma}(\bar{\varepsilon}, \boldsymbol{\xi}, \boldsymbol{x})=\boldsymbol{L}(\boldsymbol{x}): \boldsymbol{A}(\boldsymbol{x}): \overline{\boldsymbol{\varepsilon}}+\sum_{k=1}^{M} \boldsymbol{\rho}^{(k)}(\boldsymbol{x}) \xi^{(k)},
$$

where $\boldsymbol{A}$ is the elastic strain localization tensor and $\boldsymbol{\rho}^{(k)}$ are elastic fields generated by the modes $\boldsymbol{\mu}^{(k)}$. Using the decomposition (7), the effective free-energy $\widetilde{w}$ can be expressed explicitly in terms of $\bar{\varepsilon}$ and $\boldsymbol{\xi}$ (see $[7,8])$.

Expressing the effective dissipation potential $\widetilde{\varphi}$ is an even more difficult task. Fritzen and Leuschner [4] have proposed an hybrid formulation making use of the dual potential $\widetilde{\psi}=\widetilde{\varphi}^{*}$. Their definition of $\widetilde{\psi}$

$$
\widetilde{\psi}(\overline{\boldsymbol{\varepsilon}}, \boldsymbol{\xi})=\langle\psi(\boldsymbol{\sigma}(\overline{\boldsymbol{\varepsilon}}, \boldsymbol{\xi})\rangle, \quad \boldsymbol{\sigma} \text { given by }(7),
$$

is not rigorously equivalent to the exact definition by duality (as shown in [7]), but is a fairly accurate approximation of it.

But still the cost of evaluating $\langle\psi(\boldsymbol{\sigma})\rangle$ is very high, since it involves the evaluation of the local field $\boldsymbol{\sigma}(\boldsymbol{x})$ from (7), followed by the evaluation of $\psi(\boldsymbol{\sigma}(\boldsymbol{x}))$ which is a nonlinear local function of $\boldsymbol{\sigma}$, which is then averaged. An additional (and essential) step is achieved by approximating the average of the force potential $\psi$. For the sake of simplicity, attention is restricted here to polycrystals where 
the phases $V^{(r)}$ are the grains with characteristic function $\chi^{(r)}(\boldsymbol{x})$, volume fraction $c^{(r)}$ and with slip systems $\boldsymbol{m}_{s}^{(r)}$. The potential $\psi(\boldsymbol{\sigma})$ can be expressed as

$$
\psi(\boldsymbol{\sigma}, \boldsymbol{x})=\sum_{r=1}^{N} \chi^{(r)}(\boldsymbol{x}) \sum_{s=1}^{S} \psi_{s}^{(r)}\left(\tau_{s}^{(r)}(\boldsymbol{x})\right), \quad \tau_{s}^{(r)}(\boldsymbol{x})=\boldsymbol{\sigma}(\boldsymbol{x}): \boldsymbol{m}_{s}^{(r)}(\boldsymbol{x})
$$

Two approximations of $\langle\psi(\boldsymbol{\sigma})\rangle$ have been investigated so far. The first approximation consists in replacing $\psi$ by its tangent second-order expansion (TSO) in each individual phase ( $[7,8])$ :

$$
\widetilde{\psi}(\overline{\boldsymbol{\varepsilon}}, \boldsymbol{\xi}) \simeq \widetilde{\psi}_{T S O}(\overline{\boldsymbol{\varepsilon}}, \boldsymbol{\xi})=\sum_{r=1}^{N} c^{(r)} \sum_{s=1}^{S}\left[\psi_{s}^{(r)}\left(\bar{\tau}_{s}^{(r)}\right)+\frac{1}{2} \frac{\partial^{2} \psi_{s}^{(r)}}{\partial \tau^{2}}\left(\bar{\tau}_{s}^{(r)}\right) C^{(r)}\left(\tau_{s}^{(r)}\right)\right], \quad \bar{\tau}_{s}^{(r)}=\left\langle\tau_{s}^{(r)}\right\rangle^{(r)}
$$

where $C^{(r)}\left(\tau_{s}^{(r)}\right)=\left\langle\left(\tau_{s}^{(r)}-\bar{\tau}_{s}^{(r)}\right)^{2}\right\rangle^{(r)}$ denotes the quadratic fluctuations of $\tau_{s}^{(r)}$ over grain $(r)$. The second approximation was proposed in [9], based on numerical integration and following and extending on Ponte Castañeda "fully optimized" method [11]. In its simplest version it consists in replacing $\widetilde{\psi}$ by

$$
\begin{gathered}
\widetilde{\psi}_{F O}(\overline{\boldsymbol{\varepsilon}}, \boldsymbol{\xi})=\sum_{r=1}^{N} c^{(r)} \sum_{s=1}^{S} \frac{1}{2}\left(\psi_{s}^{(r)}\left(\check{\tau}_{s}^{(r)}\right)+\psi_{s}^{(r)}\left(\hat{\tau}_{s}^{(r)}\right)\right), \check{\tau}_{s}^{(r)}=\left\langle\tau_{s}^{(r)}\right\rangle^{(r)}-C^{(r)}\left(\tau_{s}^{(r)}\right), \\
\hat{\tau}_{s}^{(r)}=\left\langle\tau_{s}^{(r)}\right\rangle^{(r)}+C^{(r)}\left(\tau_{s}^{(r)}\right) .
\end{gathered}
$$

\section{Sample example: creep of polycrystalline ice}

Ice, as a single crystal, is an HCP material with 12 slip systems ( 3 basal, 3 prismatic and 6 pyramidal). The single crystal plasticity model used in the present example is that proposed in [12]. This model accounts for both kinematic and isotropic hardening. A creep test on polycrystalline ice is simulated. The representative polycrystalline aggregate used in the full-field simulations is shown in Fig. $2 \mathbf{a}$. Three full-field simulations were performed with different material data. The first data set is that identified in [12]. The other two data sets correspond to different values of the latent hardening parameters and have been chosen to highlight the sensitivity of the response to the hardening parameters ( [8]). The predictions of the two NTFA models for the overall response of the polycrystalline aggregate with these three different data sets are compared in Figs $1 \mathbf{a}$ and $1 \mathbf{b}$ with full-field simulations. The agreement between the NTFA predictions and the full-field simulations is seen to be slightly better in Fig. 1a than in Fig. 1b. This is due to the fact that the modes used in the NTFA simulations of Fig. 1b are those determined with the first set of material parameters. However, the plots in Fig. 1b show that the modes determined with the first set of material parameters can be used with other parameters and still capture correctly the influence of these parameters.

The activity of the basal slip systems, the most active ones, as found from the full-field simulation with the first set of material paramaters and as obtained by post-processing the results of the NTFA models are compared in Figs $\mathbf{2 b - d}$ at the end of the creep test. Regarding the gain in computational time, the acceleration provided by the NTFA models is of the order of $10^{2}$ for both models. The NTFA-FO model is however more appropriate for porous materials (Michel and Suquet [9]). 

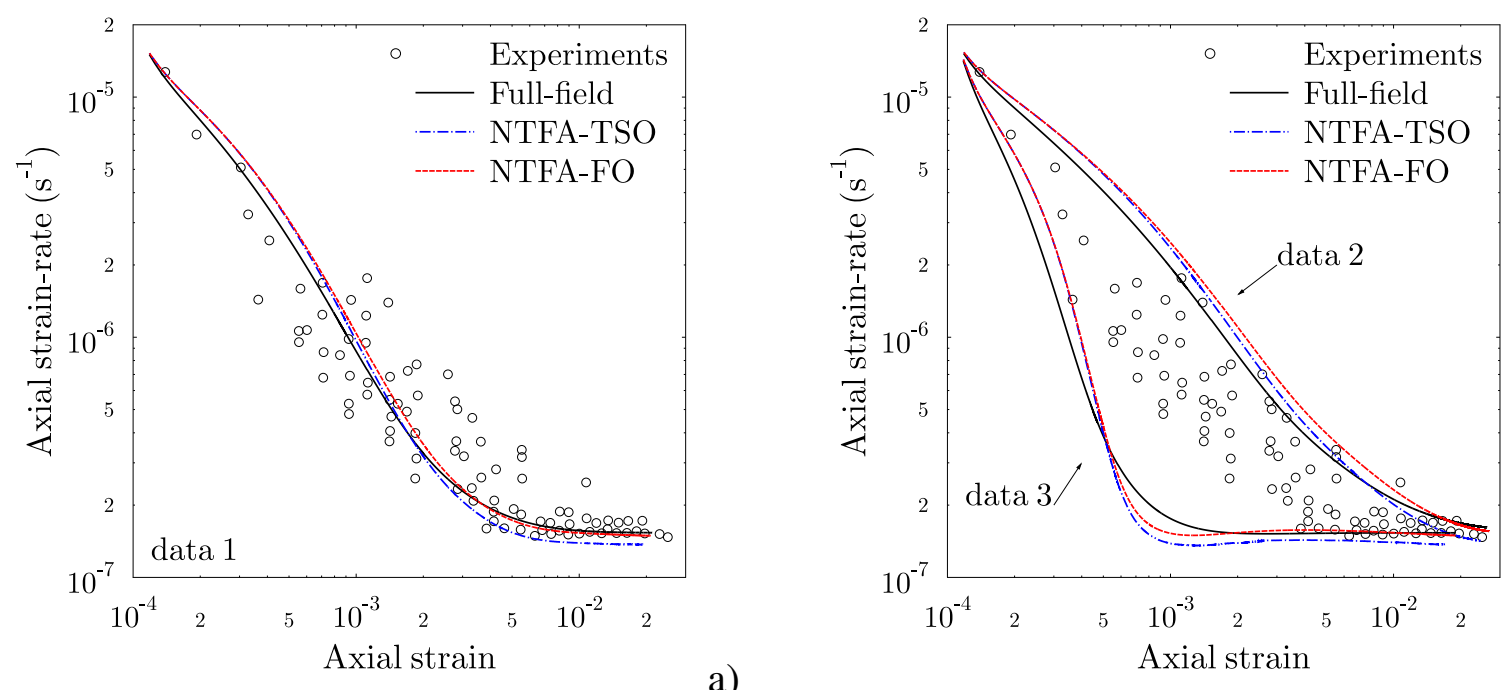

a)

b)

Figure 1: Creep of polycrystalline ice. Comparison between the full-field simulations (black solid line) and the predictions of the NTFA-TSO model (dotted-dashed blue line) and of the NTFA-FO model (dashed red line) for the response of the polycrystalline aggregate. a Material parameters identified in [12]. b Influence of the latent hardening parameters.
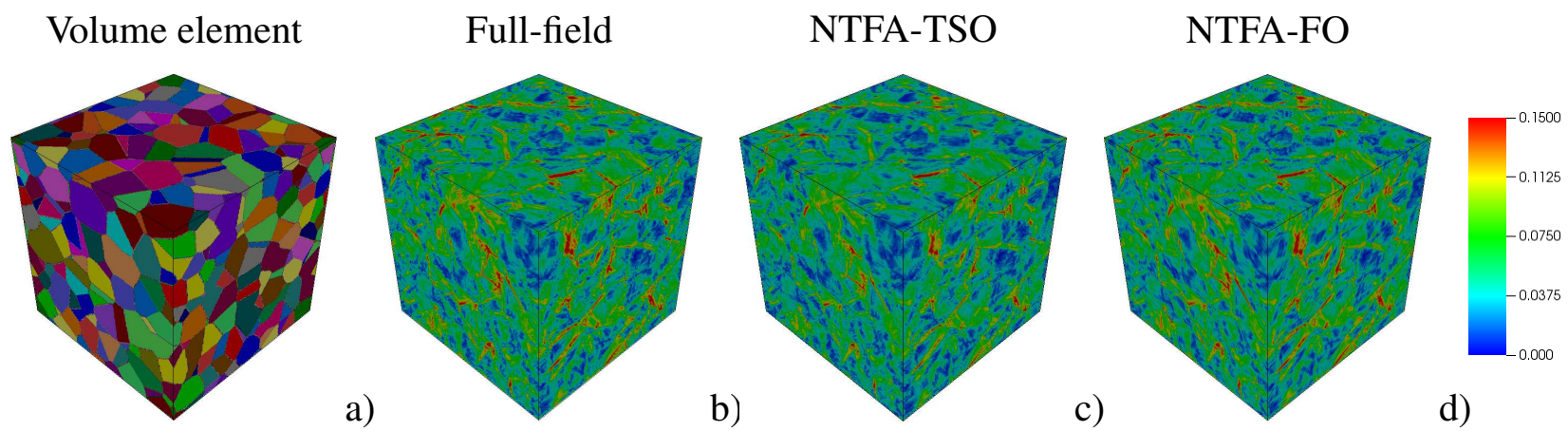

d)

Figure 2: Creep of polycrystalline ice. a Configuration with 500 grains used in the full-field simulations. b-d Snapshots of the activity of the basal slip systems at the end of the creep test. Material parameters identified in [12]. Comparison between the full-field simulation and the predictions of the NTFA models. b Full-field. c NTFA-TSO. d NTFA-FO.

\section{References}

[1] F. Chinesta, P. Ladevèze and E. Cueto, Arch. Comput. Methods Eng. 18, 395-404 (2011).

[2] D. Lucia, P. Beran and W. Silva, Progress in Aerospace Sciences 40, 51-117 (2004).

[3] J.-C. Michel and P. Suquet, Int. J. Solids Structures 40, 6937-6955 (2003). 
[4] F. Fritzen and M. Leuschner, Comput. Methods Appl. Mech. Engrg. 260, 143-154 (2013).

[5] V. Sepe, F. Auricchio, S. Marfia and E. Sacco, Comput. Mech. 57, 755-772 (2016).

[6] R. Largenton, J.-C. Michel and P. Suquet, Mech. Mat. 73, 76-100 (2014).

[7] J.-C. Michel and P. Suquet, J. Mech. Phys. Solids 90, 254-285 (2016).

[8] J.-C. Michel and P. Suquet, Comput. Mech. 57, 483-508 (2016).

[9] J.-C. Michel and P. Suquet, Proc. R. Soc. A 473:20170213 (2017).

[10] P. Ponte Castañeda and P. Suquet, In: Advances in Applied Mechanics, vol. 34, Academic Press, 171-302 (1998).

[11] P. Ponte Castañeda, Proc. R. Soc. A 471: 20150665 (2015).

[12] P. Suquet, H. Moulinec, O. Castelnau, N. Lahellec, M. Montagnat, F. Grennerat, P. Duval and R. Brenner, Procedia IUTAM 3, 76-90 (2012). 